\title{
Pollen morphology of Cactaceae in Northern Chile
}

\section{Morfología polínica de Cactáceas en el norte de Chile}

\author{
Floreana Miesen ${ }^{1 *}$, María Eugenia de Porras² \& Antonio Maldonado ${ }^{2,3}$ \\ ${ }^{1}$ Department of Geography, Rheinische Friedrich-Wilhelms-Universität Bonn, Meckenheimer Allee 166, 53115 Bonn, \\ Germany. \\ ${ }^{2}$ Centro de Estudios Avanzados en Zonas Áridas, Universidad de La Serena, Raúl Bitrán 1305, La Serena, Chile. \\ ${ }^{3}$ Departamento de Biología Marina, Universidad Católica del Norte, Larrondo 1281, Coquimbo, Chile. \\ *floreana.miesen@outlook.de
}

\begin{abstract}
Chile is habitat to over 140 species of cactus of which $45 \%$ are endemic and most of them grow in the arid northernmost part of the country between $18^{\circ}-32^{\circ} \mathrm{S}$. As the Cactaceae family plants are quite well adapted to arid environments, their fossil pollen may serve as a tool to reconstruct past environmental dynamics as well as to trace some issues regarding the family evolution or even some autoecological aspects. Aiming to create a reference atlas to be applied to some of these purposes, the pollen morphology of the following 14 different species of the Cactaceae family from Northern Chile was studied under optical microscopy: Cumulopuntia sphaerica, Maihueniopsis camachoi, Tunilla soehrensii, Echinopsis atacamensis, Echinopsis coquimbana, Haageocereus chilensis, Oreocereus hempelianus, Oreocereus leucotrichus, Copiapoa coquimbana, Eriosyce aurata, Eriosyce subgibbosa, Eulychnia breviflora, Browningia candelaris and Corryocactus brevistylus. Pollen grains of species of the subfamily Opuntioideae are spheroidal, apolar and periporate whereas grains of the subfamily Cactoideae are subspheroidal, bipolar and tricolpate and can be taxonomically differentiated between tribes. The results show that it is possible to identify pollen from the Cactaceae family at the genus level but pollen taxonomic resolution may be complicated to identify up to a specific level. A wider reference collection considering more characters than those included in the present study could improve this aspect in the near future.
\end{abstract}

KeYwords: Pollen morphology, Cactaceae, biogeography, palaeoecology, Chile.

\begin{abstract}
RESUMEN
Actualmente crecen en Chile más de 140 especies de cactus, de las cuales el 45\% son endémicas y la mayoría de ellas se distribuyen en la zona norte y más árida del país entre $18^{\circ}-32^{\circ} \mathrm{S}$. Como las Cactáceas se encuentran muy bien adaptadas a los ambientes áridos, el polen fósil de esta familia puede ser utilizado como herramienta para reconstruir la dinámica ambiental pasada así como para estudiar aspectos relacionados a su historia evolutiva o bien de su autoecología. Con el objetivo de crear una colección de referencia asociada a los propósitos mencionados anteriormente, se estudió con microscopía óptica la morfología del polen de las siguientes 14 especies de Cactáceas del norte de Chile: Cumulopuntia sphaerica, Maihueniopsis camachoi, Tunilla soehrensii, Echinopsis atacamensis, Echinopsis coquimbana, Haageocereus chilensis, Oreocereus hempelianus, Oreocereus leucotrichus, Copiapoa coquimbana, Eriosyce aurata, Eriosyce subgibbosa, Eulychnia breviflora, Browningia candelaris y Corryocactus brevistylus. Los granos de polen de las especies de la subfamilia Opuntioideae son esferoidales, apolares y periporados. Granos de la subfamilia Cactoideae son subesferoidales, isopolares y tricolpados y se pueden diferenciar taxonómicamente entre tribus. Los resultados indican que es posible diferenciar los granos de polen de las Cactáceas a nivel de género pero que debido a la resolución taxonómica de este análisis, la determinación a nivel de especie sería más complicada. Sin embargo, una colección de polen de referencia de cactáceas más amplia y considerando más caracteres que los incluidos en este trabajo podrían mejorar este problema en el futuro.
\end{abstract}

Palabras clave: Morfología polínica, Cactaceae, fitogeografía, paleoecología, Chile.

\section{INTRODUCTION}

Chile is habitat to over 140 species of cactus of which $45 \%$ are endemic. The vast majority grows in the north between
Arica and Parinacota (XV) to Coquimbo (IV) regions, from $18^{\circ}$ to $32^{\circ} \mathrm{S}$ (Moreira-Muñoz 2011, Riedemann et al. 2006) but some species may reach much further south $\left(43^{\circ} \mathrm{S}\right.$; Anderson 2001). It is not uncommon to find such a large 
number of endemic species of cactus in Northern Chile, as this area is characterized by extensive aridity and home to the Atacama, a hyper arid desert which extends along the Pacific Andean slope from southern Peru $\left(18^{\circ} \mathrm{S}\right)$ throughout Northern Chile at $27^{\circ} \mathrm{S}$ (Latorre et al. 2005). Although not restricted to these environments, species of the Cactaceae family form the characteristic vegetation of arid areas of Chile (Pinto \& Kirberg 2009). Given the prevailing unfavorable conditions in these areas, including intense solar radiation and lack of water, Cactaceae family plants are the outcome of xerophytic evolution and have especially adapted for survival in arid environments. Their most important feature is the succulence or their ability to store water (Guerrero et al. 2011, Hoffmann \& Walter 2004).

Nevertheless, ecological and climatic conditions are not uniform throughout Northern Chile (Garreaud et al. 2003, Garreaud et al. 2008, Moreira-Muñoz 2011). The Andean relief determines distinct ecological zones including coastal range, "pampa" (mostly endorheic basins), foothills and highlands. Climate is determined by atmospheric circulation patterns as well as topographic features. Thus, the Andes and the coastal cliff play main roles determining precipitation patterns. At the Northernmost part $\left(18^{\circ}-25^{\circ} \mathrm{S}\right)$ summer rainfall related to an Amazonian basin brings moisture to the Western slope of the Andes. Moisture from the Pacific Ocean is unable to reach the continent due of the combined effect of the large scale subsidence associated to the semi-permanent South Pacific Anticyclone, the altitude of the thermal inversion layer ( 800 masl) and the altitude of the coastal cliff (1000 masl) as well as the cold South-North Humboldt oceanic current along the continent coast (Garreaud et al. 2003, Garreaud et al. 2008). In the South $\left(>27^{\circ} \mathrm{S}\right)$, winter precipitation related to the Southern Westerly Wind Belt brings about a completely different precipitation pattern. Temperature patterns are highly related to the topography and negatively correlated to altitude (Garreaud et al. 2008).

In accordance to these varying environments and climates, species of the Cactaceae family similarly vary in terms of morphology and geographical distribution, showing diverging adaptations to the respective ecological and climatologic conditions (Guerrero et al. 2013). Plant and flower morphology of the Cactaceae family in Chile have been thoroughly studied in the past (Pinto \& Kirberg 2009, Hoffmann \& Walter 2004, Riedemann et al. 2006, Señoret Espinosa \& Acosta Ramos 2013). However, detailed studies of the plants' reproductive material (i.e. the pollen) or pollination ecology have not been done in northern Chile.

The comprehension and analysis of pollen morphology not only forms an essential part of any ecological research, but also serves as an important record to trace past environmental conditions, especially in arid regions where cacti are abundant (Holmgren et al. 2001, Jiménez et al. 2011, Mujica et al. 2014). As the corpuses of cacti are generally ill-suited for the fossilization process the fossil record of Cactaceae is very meager (Moreira-Muñoz 2011). In contrast, the composition of the walls of pollen grains is remarkably resistant to physical and chemical degradation, allowing the pollen to be preserved for million to thousands years under proper conditions in different environments. Various studies have shown that pollen extracted from fossil material such as lake sediments, bogs and rodent middens among other archives reflect the vegetation patterns at the examined point of time and space (Faegri \& Iversen 1989, Bennett \& Willis 2001). In Northern Chile, research has traced a considerable variability in precipitation resources related to the summer/winter precipitation sources for the last 50.000 years BP (Grosjean et al. 2001, Latorre et al.2002, 2003, Mujica et al. 2014, Maldonado et al. 2005, 2010). Considering the preeminent adaption of Cactaceae family plants to poor availability of water, their chronological occurrence in fossil records would be an indisputable indicator for hydrological conditions and change. Moreover, given that the temporal origin of the Cactaceae family and their evolution is still subject of discussion (Moreira-Muñoz 2011, Griffith 2004) fossil pollen records and identification to a (though limited but specific) taxonomic level may allow advances in this field of research.

Pollen morphology of some species of the Cactaceae family have been studied in other geographical settings throughout North Central and South America (Garralla et al. 2013, Cuadrado \& Garralla 2009, Leuenberger 1976a,b, Garralla \& Cuadrado 2007, Herrera \& Urrego 1996, Kurtz 1948, 1963).Yet to date, no pollen reference for Northern Chile has been published except for Heusser (1971) and Markgraf and D'Antoni (1978) pollen atlases which includes only four species (Opuntia ovata, Eulychnia acida, Cereus chiloensis and Maihuenia poeppigii).

The aim of this study is thus to initiate a comprehensive examination of pollen morphology of Cactaceae family in Northern Chile, beginning with 14 selected species Cumulopuntia sphaerica, Maihueniopsis camachoi, Tunilla soehrensii, Echinopsisatacamensis, Echinopsiscoquimbana, Haageocereus chilensis, Oreocereus hempelianus, Oreocereus leucotrichus, Copiapoa coquimbana, Eriosyce aurata, Eriosyce subgibbosa, Eulychnia breviflora, Browningia candelaris and Corryocactus brevistylus. The pollen description of the 14 species studied in this paper should thus serve as a tool for ecological, evolutionary and palaeoecological research.

\section{METHODS}

This study of Cactaceae pollen was based on 14 sampled flowers of Cactaceae species (Table I). Flowers were collected in the Andean zone of Tarapacá Region $\left(\sim 19^{\circ} \mathrm{S}\right)$, the coastal area of Coquimbo Region and from the Herbario of Laboratorio of Palaeoclimate and 
Palaeoecology of CEAZA (Table I). Two flowers of each species were processed in the lab following established standard procedures including $\mathrm{KOH}(10 \%)$ and acetolysis (Faegri \& Iversen1989) Pollen samples were then mounted in glycerin. Morphological characteristics of the pollen including number of units, polarity, shape, number and type of apertures as well as the description of the pollen's exine and sculpture were studied for a minimum of 10 grains each under optical microscopy under CARL ZEISS Axiostar plus microscope with $1000 \mathrm{x}$ optical zoom following the terminology of Punt et al. (2007). Further analysis and description was based on the inspection of photographs taken with Canon PowerShot A620 under the same microscope and 1000x optical zoom and also following the terminology of Punt et al. (2007).

Maps of the geographical distribution of the Cactaceae were plotted based on the information taken from various sources (Pinto \& Kirberg 2009, Hoffmann \& Walter 2004, Riedemann et al. 2006, Señoret Espinosa \& Acosta Ramos 2013) and graphically implemented in distribution maps using ArcGIS. Distribution patterns were determined mainly by latitudinal and altitudinal limits to the plants' ecological niche applied on a digital elevation model of Northern Chile, less areas of absolute desert of the Atacama. The produced maps were then revised for verification using own and external census data (Guerrero et al. 2011).

\section{RESULTS}

Subfamily Opuntioideae

Cumulopuntia sphaerica (C.F. Först.) E.F. Anderson

Monad, apolar; periporate, convex pores, 12 in number, aperture membranes resembles sculpture of grain tectum, but less dense; spheroidal, exine maximum thickness $c a$. $4 \mu$; tectate; $90-110 \mu$. Distribution: Arica and Parinacota, Tarapacá, Antofagasta, Atacama and Coquimbo Regions (Fig. 1).

Maihueniopsis camachoi (Espinosa) F. Ritter

Monad, apolar; periporate, pores oval and recessed, 12 in number, each $c a .12 \mu$ across, aperture membranes resembles sculpture of grain tectum; spheroidal; exine maximum thickness $c a$. $5 \mu$; tectate, supravaculate; 85 $110 \mu$. Distribution: Antofagasta Region, East of Calama (Fig. 2).

Tunilla soehrensii (Britton \& Rose) F. Ritter

Monad, apolar; periporate, pores recessed and oval-shaped, 12 in number, each $c a$. $10 \mu$ across; spheroidal; exine maximum thickness $c a$. $5 \mu$; tectate, verrucate, elongated sculpturing elements; $70-86 \mu$. Distribution: highlands of Arica and Parinacota Region (Fig. 3).

TABLE I. Species of the Cactaceae family studied and listed according to their taxonomic determination.

TABLA I. Especies de la familia Cactaceae estudiadas y ordenadas de acuerdo con su determinación taxonómica.

CACTACEAE

\begin{tabular}{|c|c|c|c|c|}
\hline SUBFAMILY & TRIBE & Species & COORDINATES & Site of Collection \\
\hline \multirow[t]{3}{*}{ Opuntioideae } & & Cumulopuntia spaehrica & 19¹8'65”S; 69²9’02”W & TransectoTana-Isluga \\
\hline & & Maihueniopsis camachoi & $26^{\circ} 38^{\prime} 04^{\prime \prime} \mathrm{S} ; 69^{\circ} 51^{\prime} 25^{\prime \prime} \mathrm{W}$ & Finca de Chañaral \\
\hline & & Tunilla soehrensii & $19^{\circ} 11^{\prime} 06^{\prime \prime S} ; 69^{\circ} 20^{\prime} 38^{\prime \prime} \mathrm{W}$ & Transecto Tana-Isluga \\
\hline \multirow[t]{11}{*}{ Cactoideae } & Trichocereeae & Echinopsis atacamensis & $22^{\circ} 15^{\prime} 26^{\prime \prime} \mathrm{S} ; 68^{\circ} 10^{\prime} 14^{\prime \prime} \mathrm{W}$ & Transecto Lasana-Linzor \\
\hline & & Echinopsis coquimbana & $29^{\circ} 58^{\prime} 40^{\prime \prime} \mathrm{S} ; 71^{\circ} 22^{\prime} 12^{\prime \prime} \mathrm{W}$ & El Panul, Coquimbo \\
\hline & & Haageocereus chilensis & $19^{\circ} 17^{\prime} 74^{\prime \prime} \mathrm{S} ; 69^{\circ} 28^{\prime} 05^{\prime \prime} \mathrm{W}$ & Transecto Tana-Isluga \\
\hline & & Oreocereus hempelianus & $19^{\circ} 03{ }^{\prime} 51^{\prime \prime} \mathrm{S} ; 69^{\circ} 14^{\prime} 65^{\prime \prime} \mathrm{W}$ & Transecto Tana-Isluga \\
\hline & & Oreocereus leucotrichus & $19^{\circ} 14^{\prime} 84^{\prime \prime} \mathrm{S} ; 69^{\circ} 22^{\prime} 34^{\prime \prime} \mathrm{W}$ & Transecto Tana-Isluga \\
\hline & Notocactaceae & Copiapoa coquimbana & $29^{\circ} 58^{\prime} 33^{\prime \prime} \mathrm{S} ; 71^{\circ} 22^{\prime} 10^{\prime \prime} \mathrm{W}$ & El Panul, Coquimbo \\
\hline & & Eriosyce aurata & $29^{\circ} 28^{\prime} 26^{\prime \prime} \mathrm{S} ; 70^{\circ} 38^{\prime} 59^{\prime \prime} \mathrm{W}$ & Transecto Tana-Isluga \\
\hline & & Eriosyce subgibbosa & $29^{\circ} 58^{\prime} 40^{\prime \prime} \mathrm{S} ; 71^{\circ} 22^{\prime} 12^{\prime \prime} \mathrm{W}$ & El Panul, Coquimbo \\
\hline & & Eulychnia breviflora & 29॰58’39”S; 71²2’19”W & El Panul, Coquimbo \\
\hline & Browningieae & Browningia candelaris & 19¹8'93”S; 69³0’04”W & Transecto Tana-Isluga \\
\hline & Pachycereeae & Corryocactus brevistylus & $19^{\circ} 18^{\prime} 65^{\prime \prime} \mathrm{S} ; 69^{\circ} 29^{\prime} 02^{\prime \prime} \mathrm{W}$ & Transecto Tana-Isluga \\
\hline
\end{tabular}




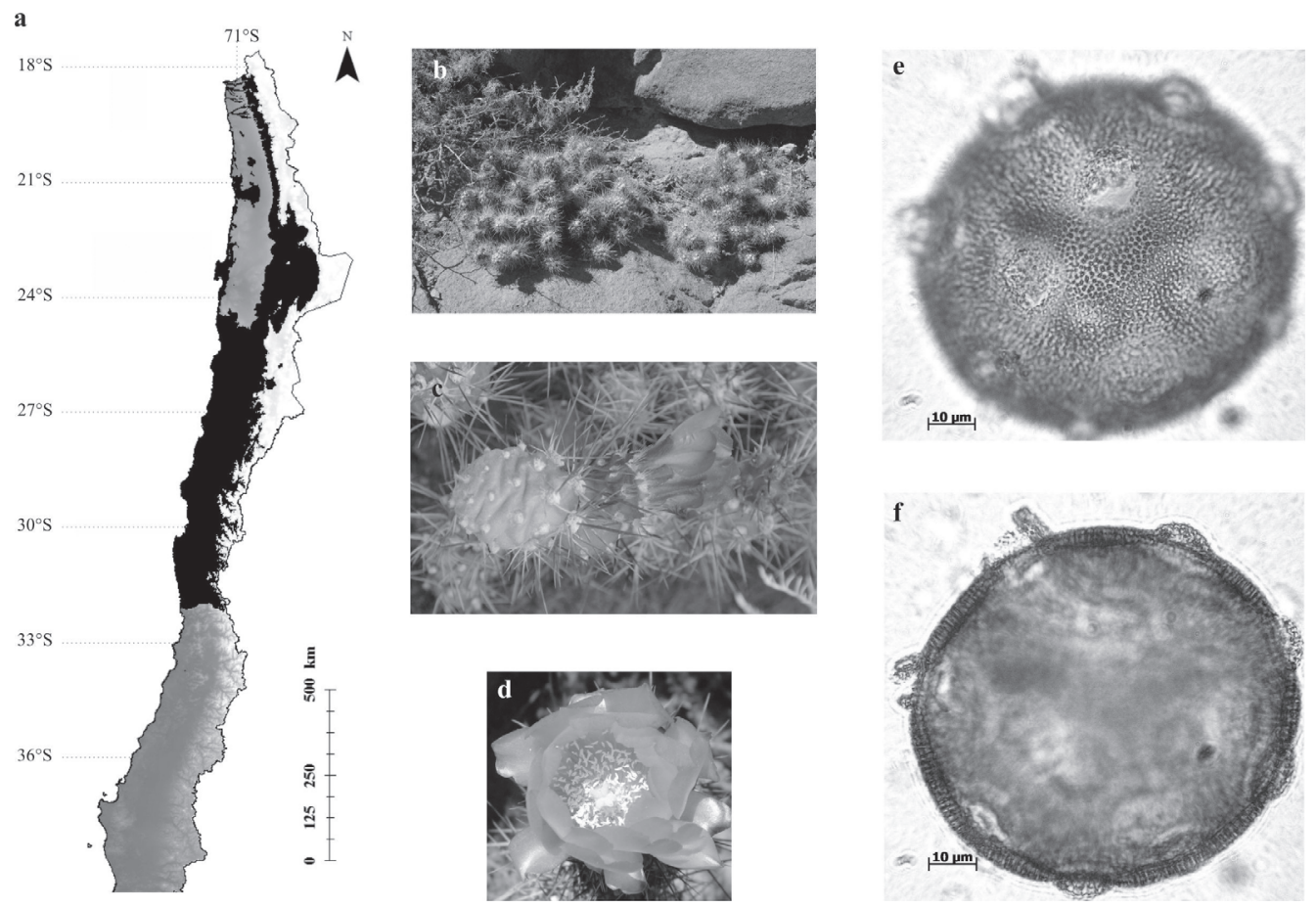

Figure 1. Cumulopuntia sphaerica distribution map (a), plant and flower photographs (b, c, d) and pollen grain photographs (e, f).

FigurA. 1. Mapa mostrando la distribución de Cumulopuntia sphaerica (a), fotografías de la planta y las flores (b, c, d) y del grano de polen $(\mathrm{e}, \mathrm{f})$.
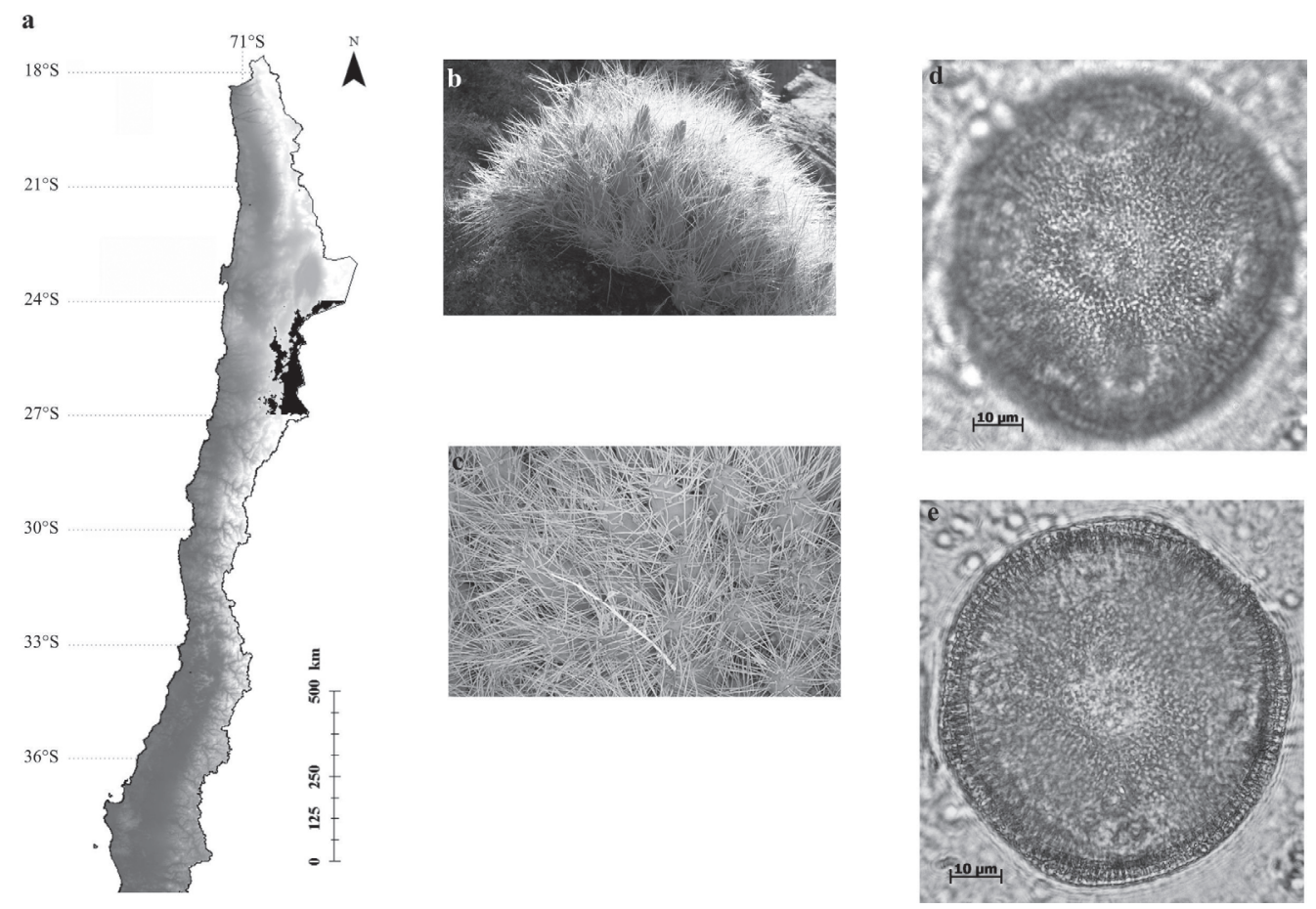

FIGURE 2. Maihueniopsis camachoi distribution map (a), plant (b, c) and pollen grain photographs (d, e).

Figura 2. Mapa mostrando la distribución de Maihueniopsis camachoi (a), fotografías de la planta (b, c) y del grano de polen (d, e). 

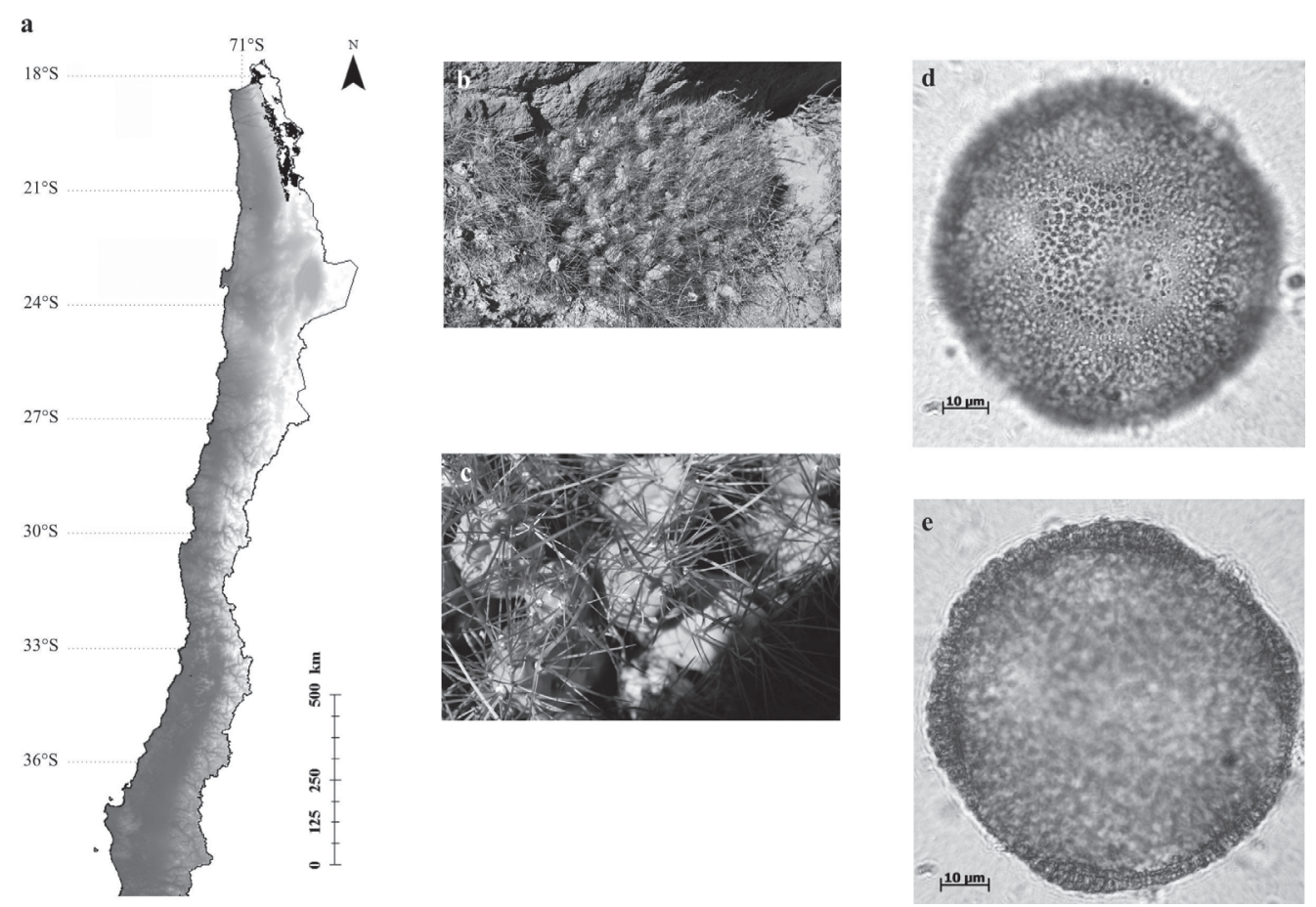

FIGURE 3. Tunilla soehrensii distribution map (a), plant (b, c) and pollen grain photographs (d, e).

FiguRA 3. Mapa mostrando la distribución de Tunilla soehrensii (a), fotografías de la planta (b, c) y del grano de polen (d, e).

Subfamily Cactoideae

TRIBE TrichocereEAE

Echinopsis atacamensis (Phil.) Friedrich \& Rowley

Monad, isopolar, tricolpate, colpi narrow, triangular and straight, sculptured, each $c a$. $20 \mu$ in depth and $4 \mu$ across,


exine maximum thickness $c a$. $4 \mu$; tectate, foveolate, microequinate, microspines randomly distributed; $56-68 \mu$ equatorial and $52-73 \mu$ polar size. Distribution: Antofagasta Region, particularly around San Pedro de Atacama (Fig. 4).

Echinopsis coquimbana (Molina) Friedrich \& Rowley Monad, isopolar; tricolpate, colpi long and narrow, triangular and straight, pointed at tip, each $c a .23 \mu$ in depth and $c a .5 \mu$ across, mesocolpium $c a .47 \mu$, apocolpium $c a$. $18 \mu$ oblate; exine maximum thickness $c a$. $2 \mu$; tectate, foveolate, microequinate, microspines equidistantly distributed; 49$54 \mu$ equatorial and $61-66 \mu$ polar size. Distribution: Coast of Coquimbo Region (Fig. 5).

\section{Haageocereus chilensis (Meyen) F. Ritter}

Monad, isopolar; tricolpate, colpi very profound, triangular and straight, pointed at tip, moderate in width each $c a$. $25 \mu$ in depth and $6 \mu$ across, core exine microequinate,

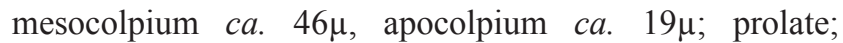
exine maximum thickness $c a$. $3 \mu$, tectate, perforated, microequinate, microspines randomly distributed; $50-58 \mu$ equatorial and $60-72 \mu$ polar size. Distribution: Arica and Parinacota Region (Fig. 6).

\section{Oreocereus hempelianus (Gürke) D.R. Hunt}

Monad, isopolar; tricolpate, colpi exine resembles sculpture of grain tectum, moderate in width, each $c a .12 \mu$ in depth and $c a .4 \mu$ across, mesocolpium $c a$. $45 \mu$, apocolpium $c a$. $24 \mu$; spheroidal; exine maximum thickness $c a .3 \mu$; tectate, foveolate, microequinate, microspines randomly distributed; 43-59 $\mu$ equatorial and 49-65 $\mu$ polar size. Distribution: Arica and Parinacota Region (Fig. 7).

Oreocereus leucotrichus (Phil.) Wagenkn. ex F. Ritter Monad, isopolar; tricolpate, colpi very similar to Oreocereus hempelianus, but more profound, each $c a .21 \mu$ in depth and $c a .5 \mu$ across, mesocolpium $c a$. $51 \mu$, apocolpium $c a .17 \mu$; spheroidal; exine maximum thickness $c a$. $3 \mu$; tectate, fossulate-foveolate, microequinate, microspines equidistantly distributed; $58-63 \mu$ equatorial and $58-73 \mu$ polar size. Distribution: Arica and Parinacota, Tarapacá and Antofagasta Regions (Fig. 8). 

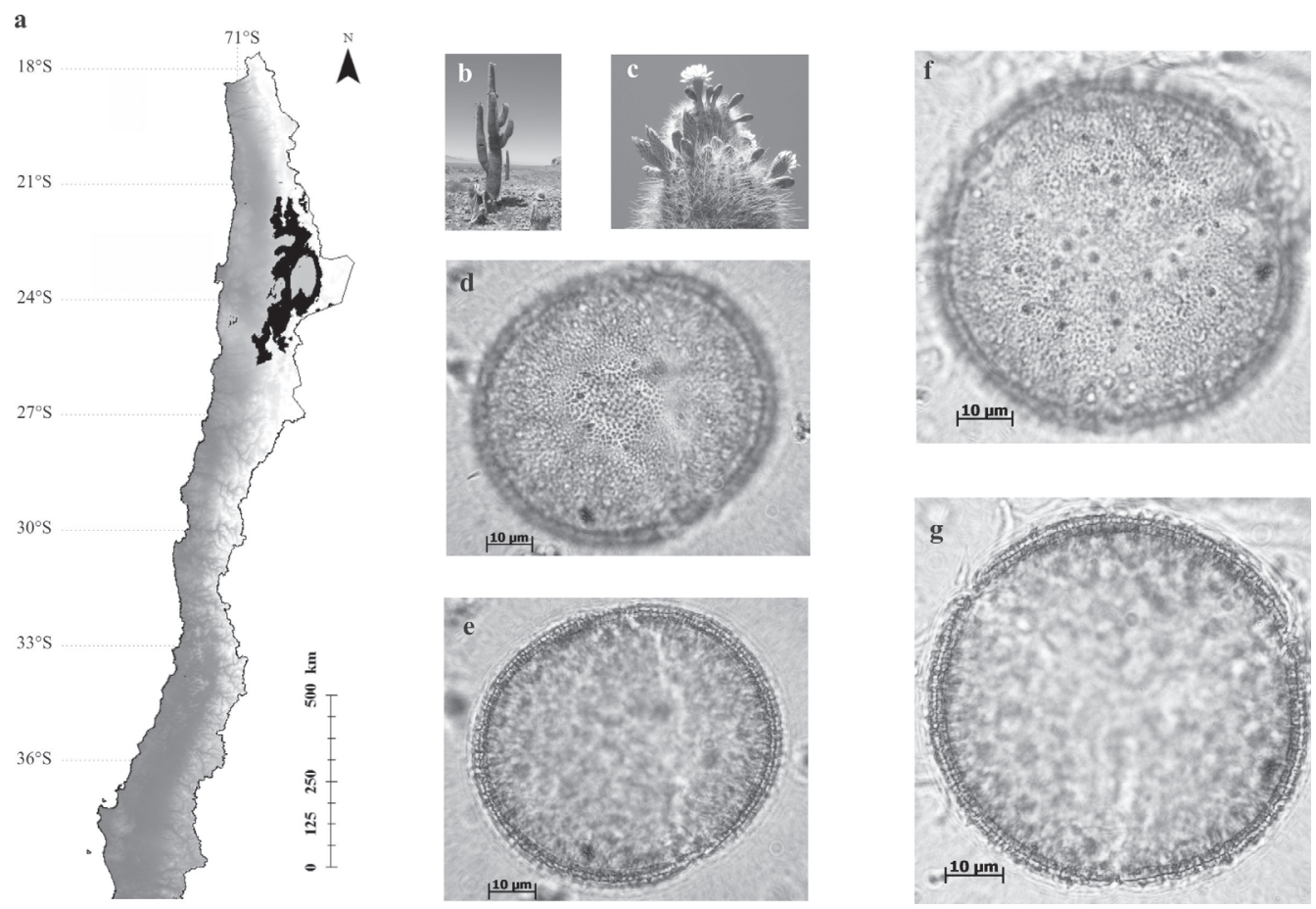

FIGURE 4. Echinopsis atacamensis distribution map (a), plant and flower photographs (b, c) and pollen grain photographs in equatorial (d, e) and polar (f, g) views.

Figura 4. Mapa mostrando la distribución de Echinopsis atacamensis (a), fotografías de la planta y las flores (b, c) y del grano de polen en vista ecuatorial $(d, e)$ y polar $(f, g)$.
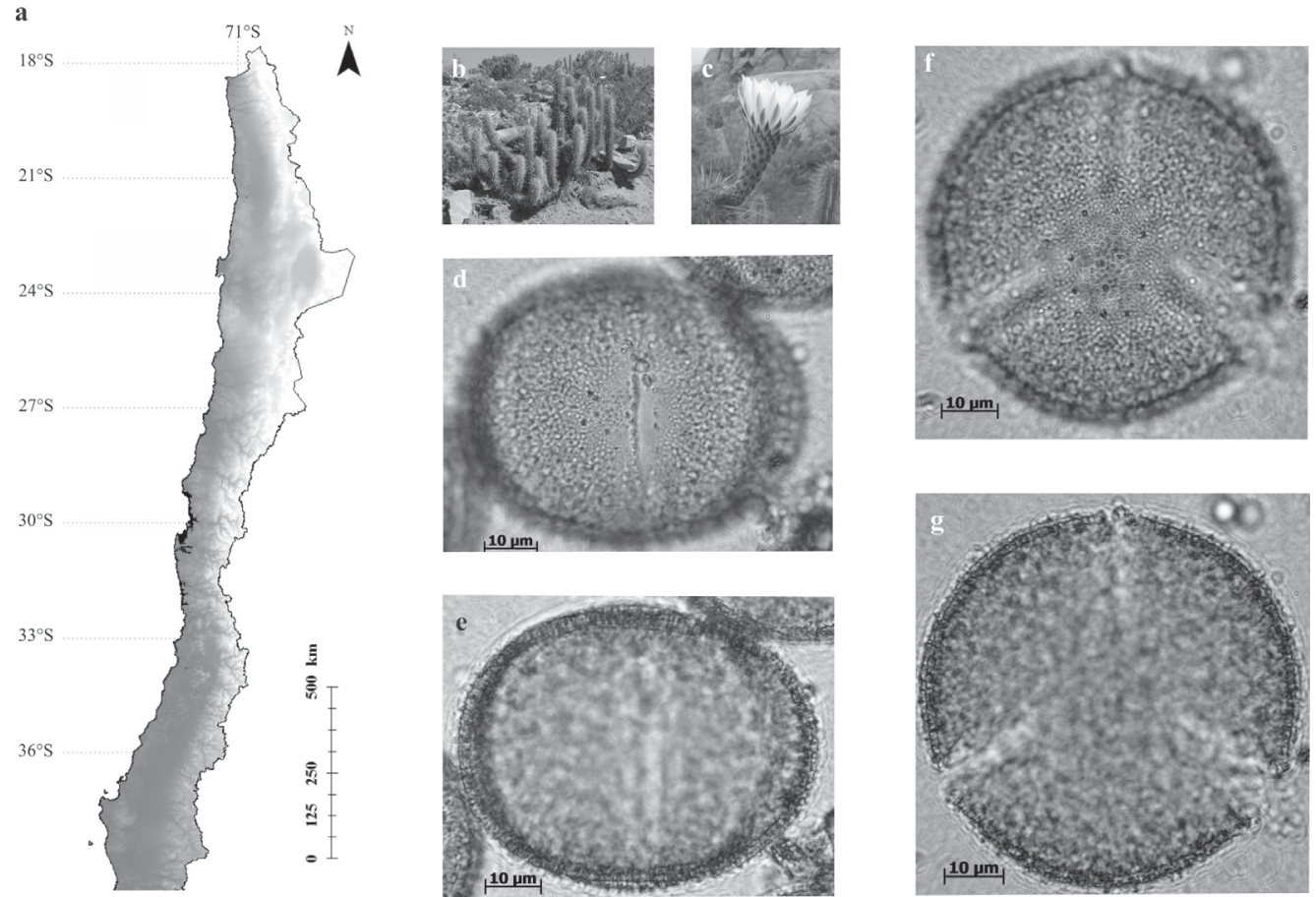

FIGURE 5. Echinopsis coquimbana distribution map (a), plant and flower photographs (b, c) and pollen grain photographs (d, e) equatorial view, (f, g) polar view.

FIGURA 5. Mapa mostrando la distribución de Echinopsis coquimbana (a), fotografías de la planta y las flores (b, c) y del grano de polen en vista ecuatorial $(\mathrm{d}, \mathrm{e})$ y polar $(\mathrm{f}, \mathrm{g})$. 



FigURE 6. Haageocereus chilensis distribution map (a), plant (b, c) and pollen grain photographs (d, e) equatorial view, (f, g) polar view.

Figura 6. Mapa mostrando la distribución de Haageocereus chilensis (a), fotografías de la planta (b, c) y del grano de polen en vista ecuatorial (d, e) y polar (f, g).
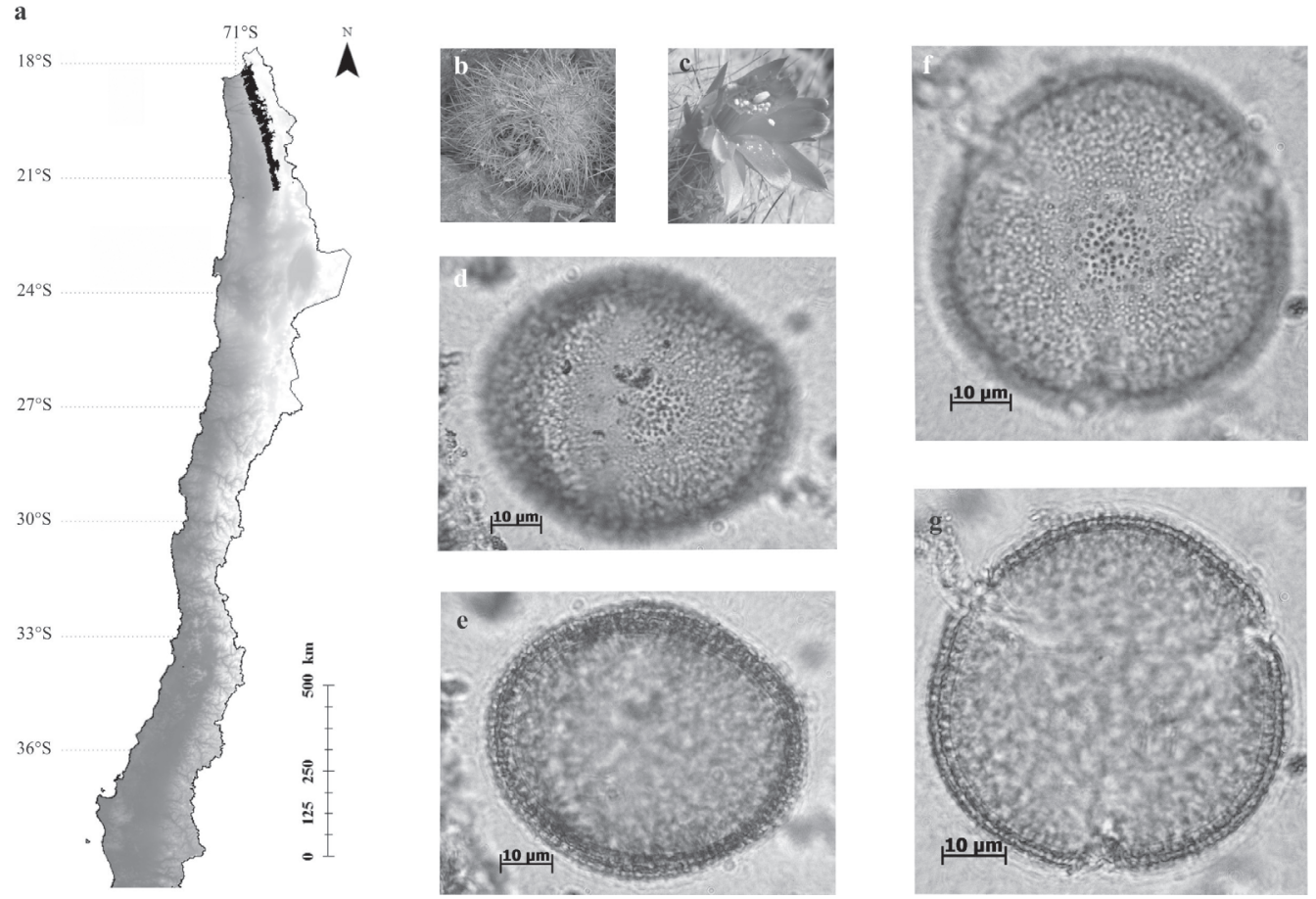

FIGURE 7. Oreocereus hempelianus distribution map (a), plant and flower photographs (b, c) and pollen grain photographs (d, e) equatorial view, (f, g) polar view.

Figura 7. Mapa mostrando la distribución de Oreocereus hempelianus (a), fotografías de la planta y las flores (b, c) y del grano de polen en vista ecuatorial $(d, e)$ y polar (f, g). 

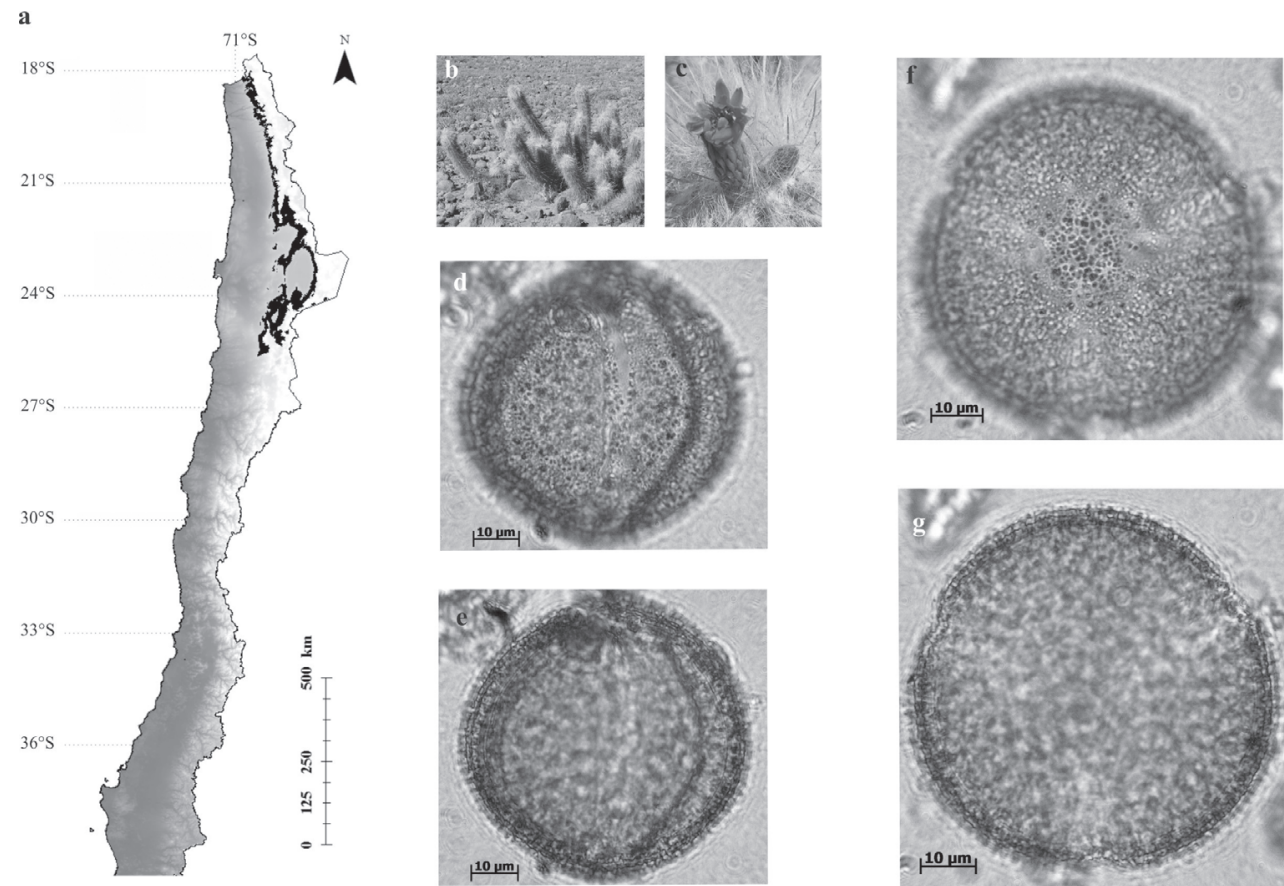

Figure 8. Oreocereus leucotrichus distribution map (a), plant and flower photographs (b, c) and pollen grain photographs (d, e) equatorial view, (f, g) polar view.

Figura 8. Mapa mostrando la distribución de Oreocereus leucotrichus (a), fotografías de la planta y las flores (b, c) y del grano de polen en vista ecuatorial $(d, e)$ y polar (f, g).

\section{Tribe Notocactaceae}

Copiapoa coquimbana (Rümpler) Britton \& Rose

Monad, isopolar; angulaperturate; tricolpate, colpi short (brevicolpate) and expanded, each $c a .17 \mu$ in depth and $c a$. $6.5 \mu$ across, aperture membrane resembles sculpture of grain tectum but much finer, mesocolpium $c a$. $44 \mu$; apocolpium ca. $19 \mu$; oblate; exine maximum thickness ca. $3 \mu$; tectate, foveolate with microspines; $43-51 \mu$ equatorial and $53-68 \mu$ polar size. Distribution: endemic to Atacama and Coquimbo Regions, close to the coast (Fig. 9).

Eriosyce aurata (Pfeiff.) Backeb.

Monad, isopolar; angulaperturate; tricolpate, brevicolpate with aperture membrane that resembles the sculpture of grain tectum but much finer, rounded at tip, each $c a .15 \mu$ in depth and $c a .6 \mu$ across, mesocolpium $c a$. $51 \mu$, apocolpium ca. $20 \mu$; oblate; exine maximum thickness $c a$. $3 \mu$; tectate, foveolate-fossulate; $47-52 \mu$ equatorial and $52-61 \mu$ polar size. Distribution: from Atacama to Metropolitana Regions (Fig. 10).

\section{Eriosyce subgibbosa (Haworth) Katterman}

Monad, isopolar; planaperturate; tricolpate, brevicolpate, colpi rounded at tip, aperture membrane resembles sculpture of grain tectum but much finer, each $c a$. $10 \mu$ in depth and $c a$. $5 \mu$ across, mesocolpium $c a .41 \mu$, apocolpium ca. $24 \mu$; oblate; exine maximum thickness ca. $2 \mu$; tectate, fossulate; $43-50 \mu$ equatorial and $52-$ $62 \mu$ polar size. Distribution: Coastal areas of Coquimbo throughout Biobío Region (Fig. 11).
Eulychnia breviflora Phil.

Monad, isopolar, tricolpate, colpi large with a sparsely sculptured aperture membrane, each $c a$. $16 \mu$ in depth and $c a$. $4 \mu$ across, mesocolpium $c a$. $42 \mu$, apocolpium $c a$. $13 \mu$; oblate; exine maximum thickness $c a$. $3 \mu$; tectate, supravaculate; 40$52 \mu$ equatorial and $50-70 \mu$ polar size. Distribution: Atacama and Coquimbo Regions (Fig. 12).

Tribe BrowningIEAE

Browningia candelaris (Meyen) Britton \& Rose

Monad, isopolar; tricolpate, expanded and moderately profound colpi, each $c a$. $17 \mu$ in depth and $c a .8 \mu$ across, mesocolpium $c a$. $74 \mu$, apocolpium $c a$. $22 \mu$; oblate; exine maximum thickness $c a$. $3 \mu$; tectate, perforated; microequinate, microspines approximately equidistantly distributed; $50-55 \mu$ equatorial and $63-67 \mu$ polar size. Distribution: Arica and Parinacota and Tarapacá Regions (Fig. 13).

\section{Tribe Pachycereeae}

Corryocactus brevistylus (K. Schum. ex Vaupel) Britton \& Rose

Monad, isopolar; tricolpate, brevicolpate and colpi expanded with rounded cones, each $c a .18 \mu$ in depth and ca. $10 \mu$ across, their exines microequinate, mesocolpium ca. $53 \mu$, apocolpium ca. $18 \mu$; subspheroidal; exine thickness maximum $c a$. $3 \mu$; tectate, foveolate, very finely microequinate (delicate microspines); $63-67 \mu$ equatorial and $52-75 \mu$ polar size. Distribution: Arica and Parinacota and Tarapacá Regions (Fig. 14). 

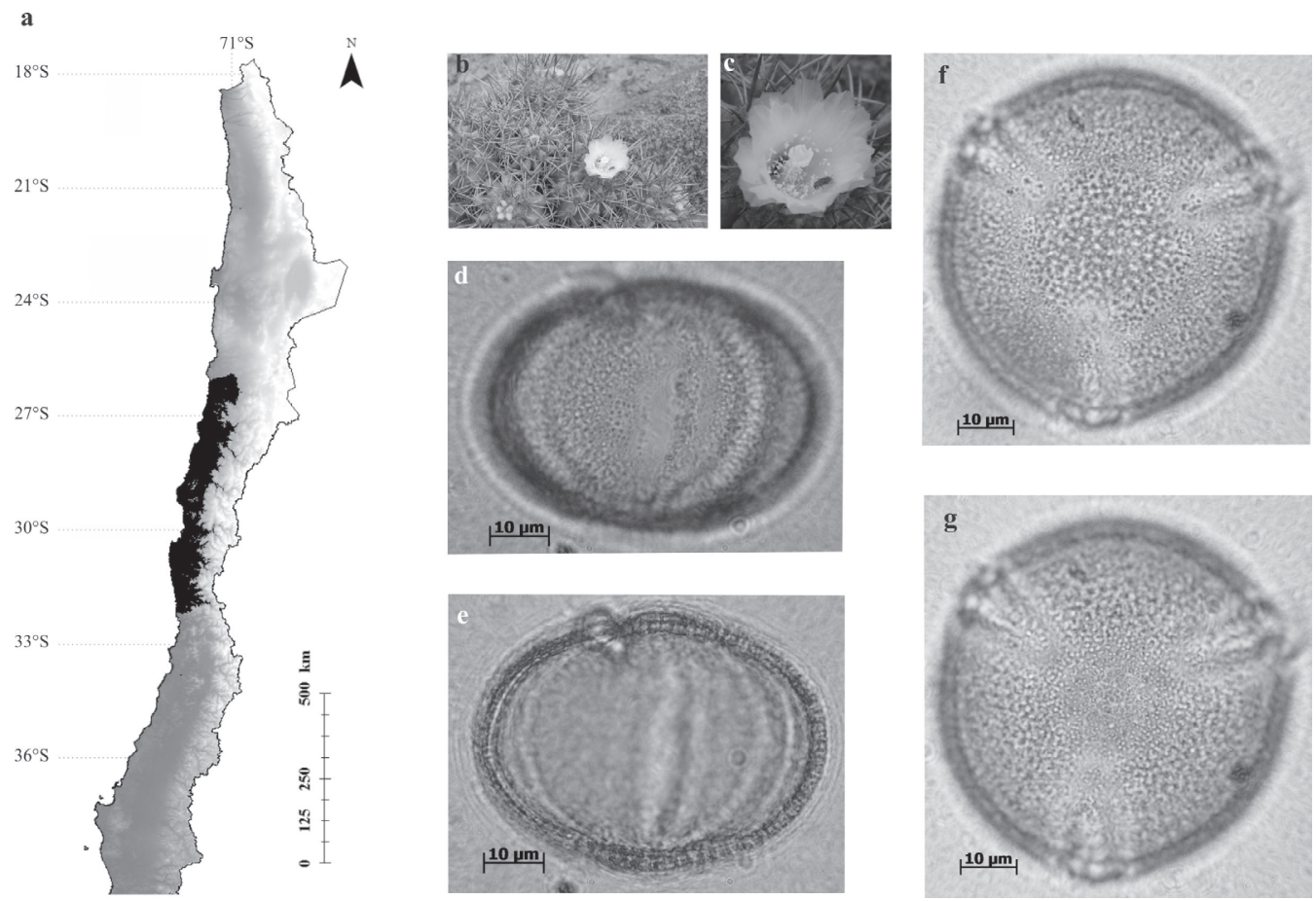

Figure 9. Copiapoa coquimbana distribution map (a), plant and flower photographs (b, c) and pollen grain photographs (d, e) equatorial view, (f, g) polar view.

Figura 9. Mapa mostrando la distribución de Copiapoa coquimbana (a), fotografías de la planta y las flores (b, c) y del grano de polen en vista ecuatorial $(\mathrm{d}, \mathrm{e})$ y polar $(\mathrm{f}, \mathrm{g})$.
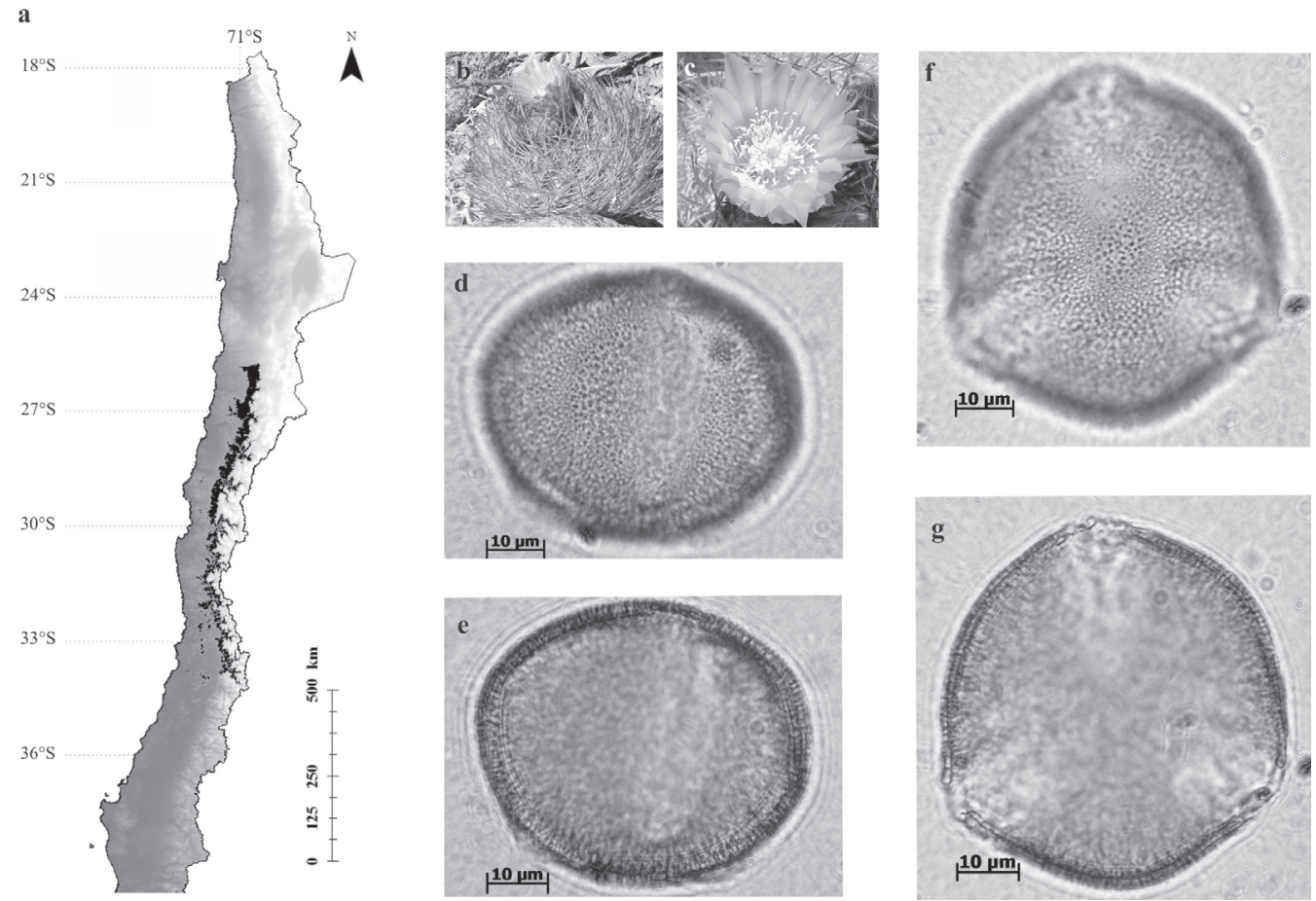

FIGURE 10. Eriosyce aurata distribution map (a), plant and flower photographs (b, c) and pollen grain photographs (d, e) equatorial view, (f, g) polar view.

FIGURA 10. Mapa mostrando la distribución de Eriosyce aurata (a), fotografías de la planta y las flores (b, c) y del grano de polen en vista ecuatorial (d, e) y polar (f, g). 

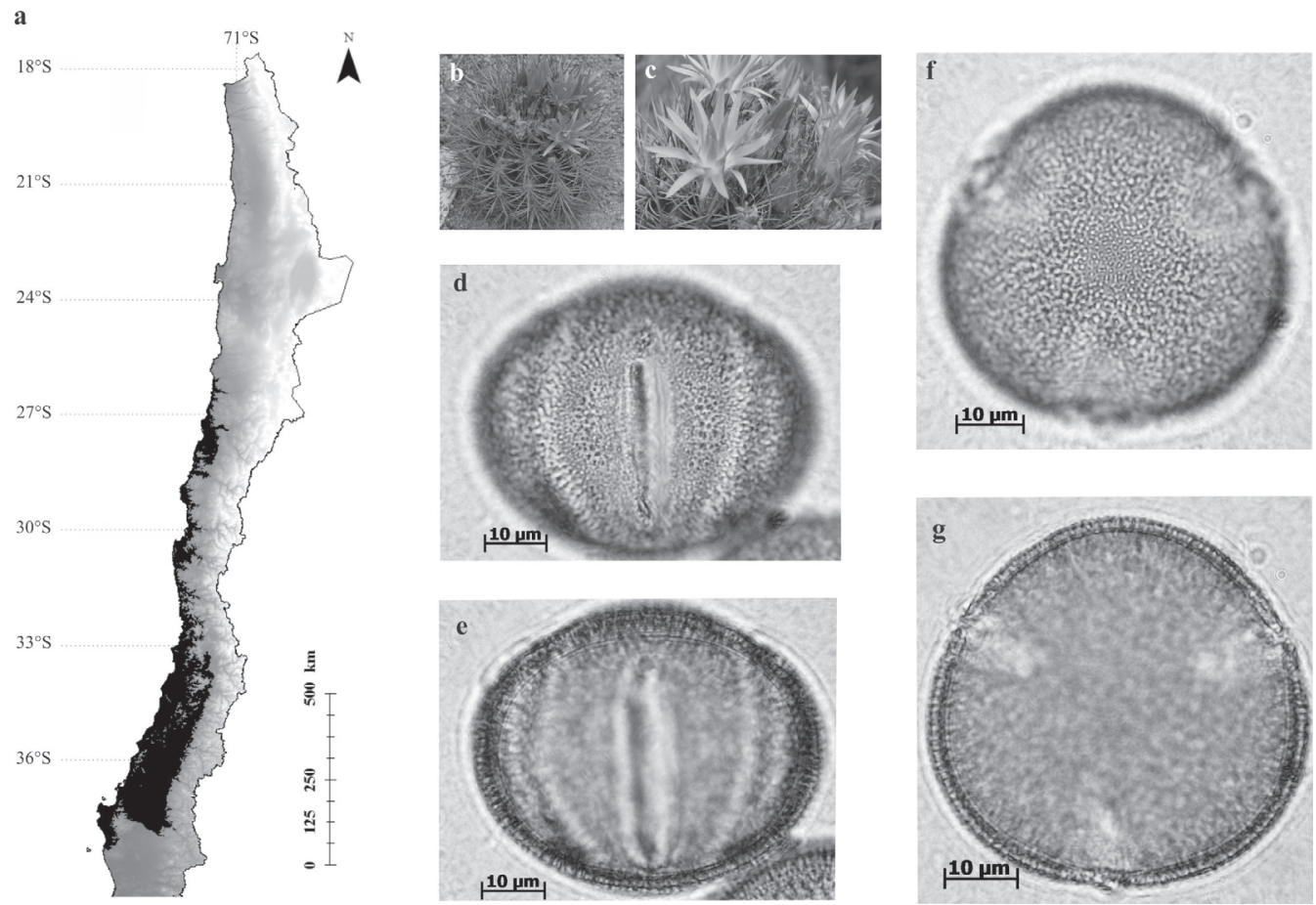

FiguRE 11. Eriosyce subgibbosa distribution map (a), plant and flower photographs (b, c) and pollen grain photographs (d, e) equatorial view, $(f, g)$ polar view.

FiguRa 11. Mapa mostrando la distribución de Eriosyce subgibbosa (a), fotografías de la planta y las flores (b, c) y del grano de polen en vista ecuatorial $(\mathrm{d}, \mathrm{e})$ y polar $(\mathrm{f}, \mathrm{g})$.

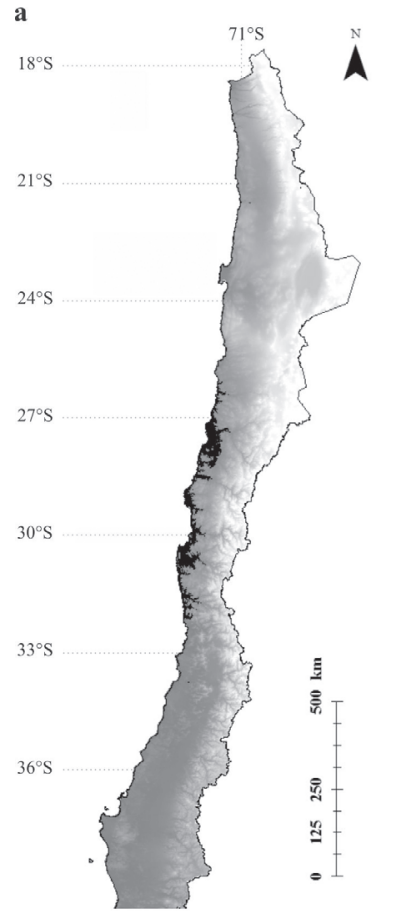

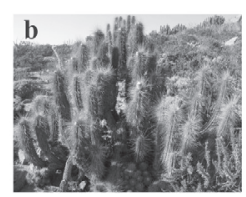
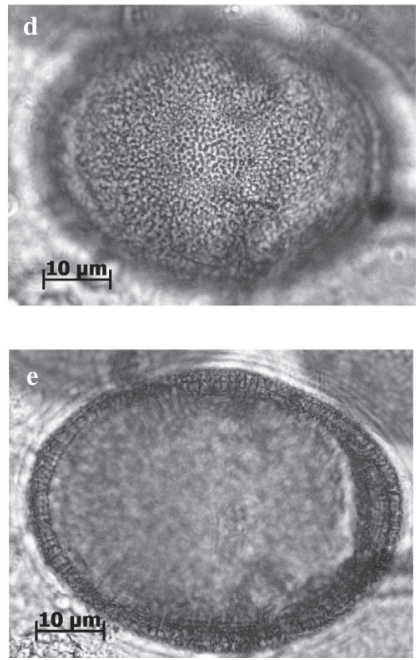
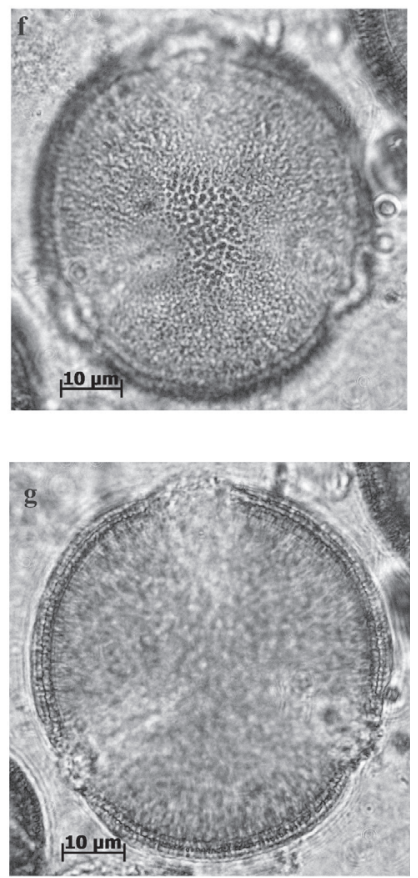

FIGURE 12. Eulychnia breviflora distribution map (a), plant and flower photographs (b, c) and pollen grain photographs (d, e) equatorial view, (f, g) polar view.

FIGURA 12. Mapa mostrando la distribución de Eulychnia breviflora (a), fotografías de la planta y las flores (b, c) y del grano de polen en vista ecuatorial $(\mathrm{d}, \mathrm{e})$ y polar $(\mathrm{f}, \mathrm{g})$. 

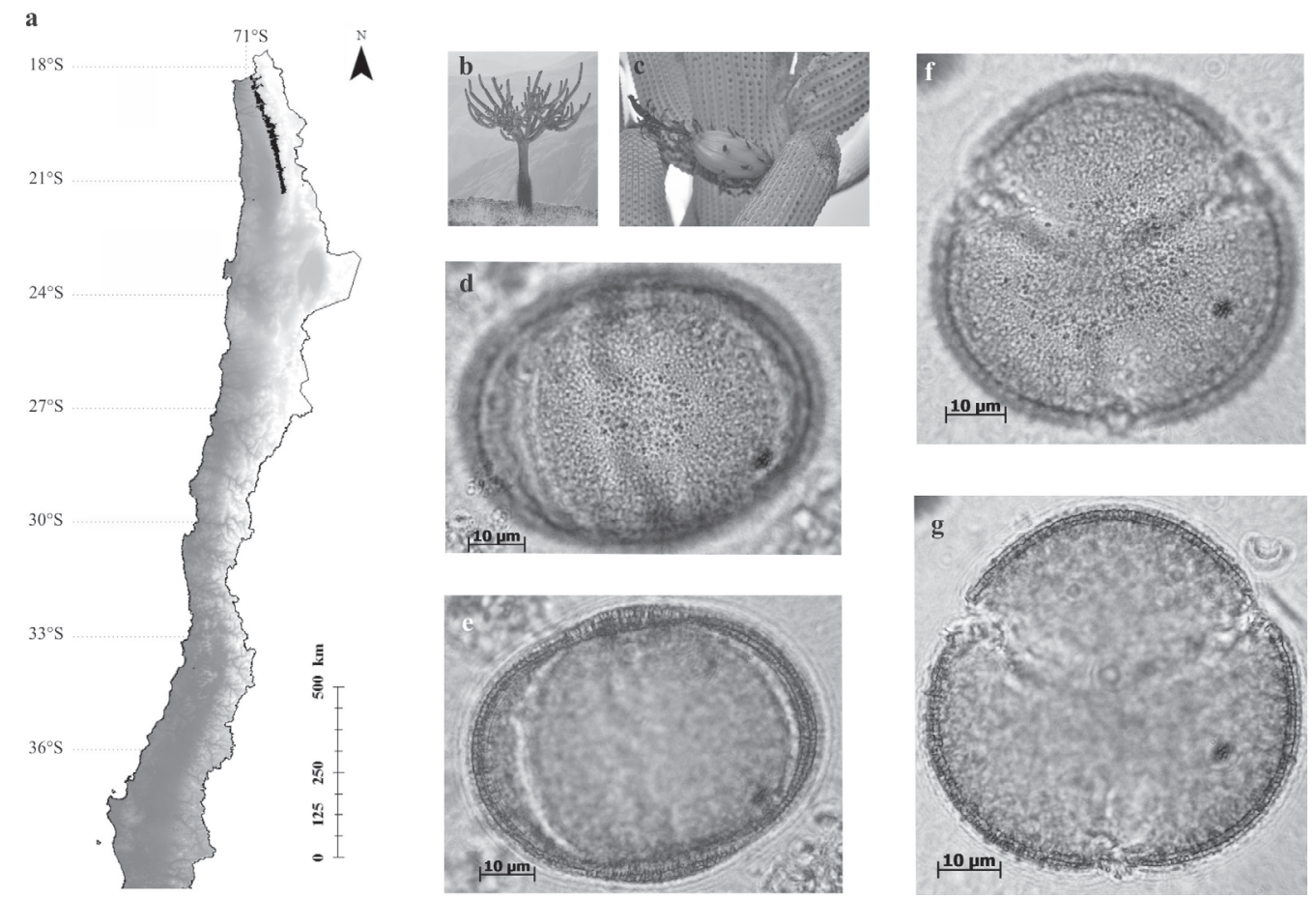

FiguRE 13. Browningia candelaris distribution map (a), plant and fruit photographs (b, c) and pollen grain photographs (d, e) equatorial view, (f, g) polar view.

Figura 13. Mapa mostrando la distribución de Browningia candelaris (a), fotografías de la planta y el fruto (b, c) y del grano de polen en vista ecuatorial $(d, e)$ y polar $(f, g)$.
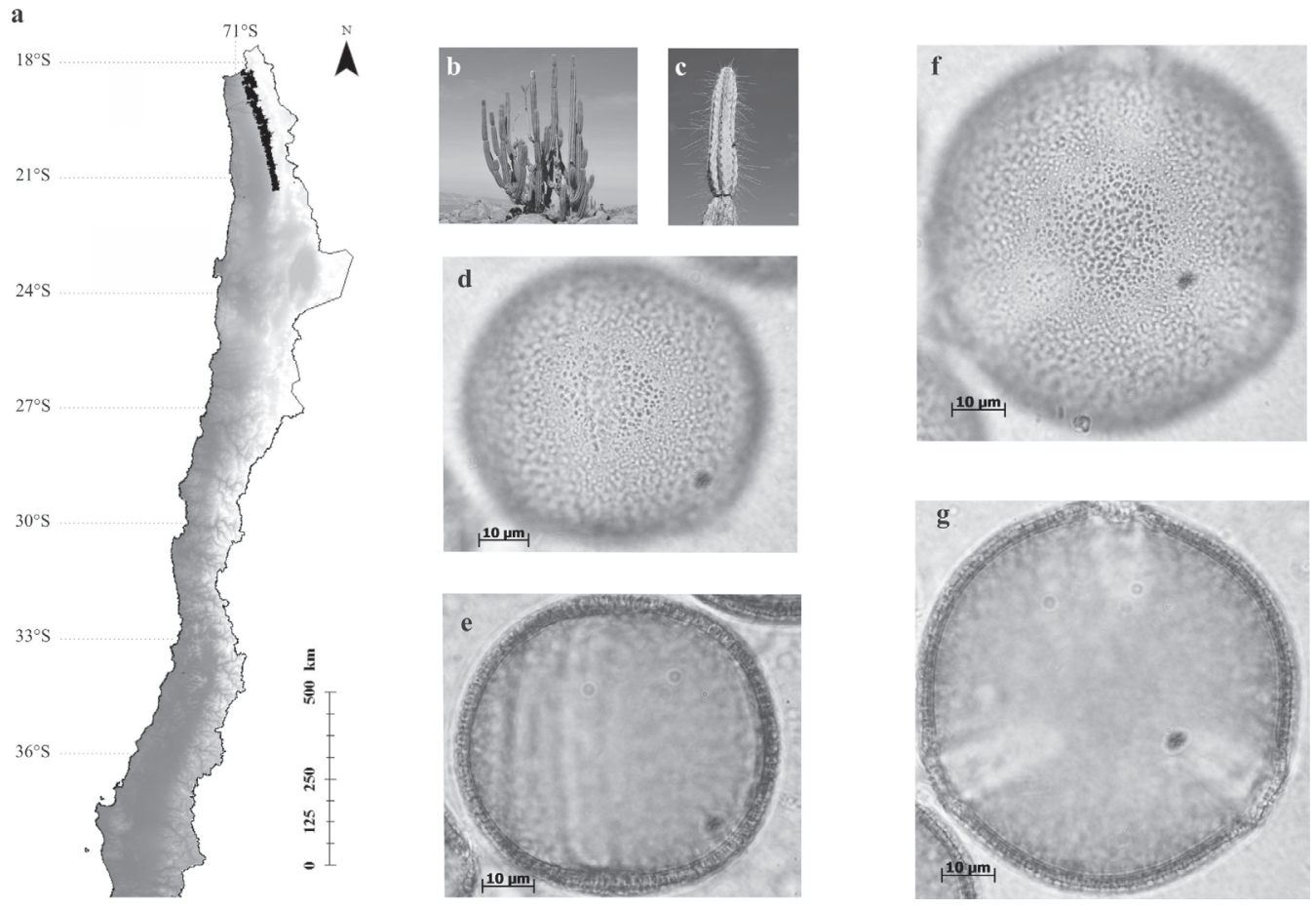

Figure 14. Corryocactus brevistylus distribution map (a), plant (b, c) and pollen grain photographs (d, e) equatorial view, (f, g) polar view.

Figura 14. Mapa mostrando la distribución de Corryocactus brevistylus (a), fotografías de la planta (b, c) y del grano de polen en vista ecuatorial $(\mathrm{d}, \mathrm{e})$ y polar $(\mathrm{f}, \mathrm{g})$. 


\section{DISCUSSION}

Pollen grains of the selected Chilean species of the Cactaceae family are subspheroidal, prolate or oblate and appear as either apolar or isopolar. Grains are either tricolpate or periporate, which still seem to be the only two forms within the Cactaceae family, as already shown in earlier studies for other geographical regions throughout the Americas (Garralla et al. 2013, Cuadrado \& Garralla 2009, Garralla \& Cuadrado 2007, Kurtz 1948, 1963). The family is further characterized by relatively large pollen grain diameter, between 40 and $100 \mu \mathrm{m}$, compared to other species in the same geographical setting $(10-45 \mu \mathrm{m})$. However, the different species cannot be distinguished by size alone, as their sizes overlap. In fact, the grains of the species studied show great morphological similarities among which might impede detailed taxonomical distinction in subsequent studies of unsorted samples of pollen.

Nevertheless, the most accessible distinction can be observed between the subfamilies. Cumulopuntia sphaerica, Maihueniopsis camachoi and Tunilla soehrensii (Fig.1-Fig.3) of the subfamily Opuntioideae are set apart by pores and different wall sculpture. Species of Opuntioideae may be easier to determine, as their pores and exines are well-defined among the subfamily. On the other hand, all species of the subfamily Cactoideae, including Browningia candelaris, Copiapoa coquimbana, Corryocactus brevistylus, Echinopsis atacamensis, Echinopsis coquimbana, Eulychnia breviflora, Eriosyce aurata, Haageocereus chilensis, Eriosyce subgibbosa, Oreocereus hempelianus and Oreocereus leucotrichus (Fig.4-Fig.14) have three colpi and a microequinate sculpture.
Concerning the subfamily Cactoideae, the grains' outline may serve as an indicator when identifying species. Grains of the tribe Trichocereeae (Fig. 4-Fig. 8) are generally characterized by a well circular outline in polar view, whereas grains of the tribe Notocactaceae (Fig.9-Fig.12) have a more angular outline, which may be angulaperturate or planaperturate, depending on the genus. The grains studied of the tribe Browningieae (Fig.13) are crescentic, with a more pronounced exine in the medium of the intercolpium, gradually thinning towards the colpi. Finally, the tribe Pachycereeae (Fig.14) may be distinguished by a compressed-elliptical outline in equatorial view, but this remains open to verification as just one species of this tribe was examined in this study.

In order to distinguish between species - especially of the subfamily Cactoideae- in pollen samples, special attention should thus be paid to the detailed study of the grains' size, outline and shape of the apertures as well as the pollen grain wall sculpture. The key to the pollen of studied species of northern Chile provided here may serve as a tool to identify these species (see end of discussion section).

The results highlight the importance of developing pollen reference collections of the Cactaceae family in northern Chile to be applied to ecological, palaeoecological and evolutionary research. However, as recorded for other families such as Asteraceae or Poaceae, pollen taxonomic resolution may be complicated to identify up to the specific level within Cactaceae family but a wider reference collection considering more characters than those included in the present study could improve this aspect in the near future.

Key to the POllen OF STUdied SPECIES OF NORTHERn Chile

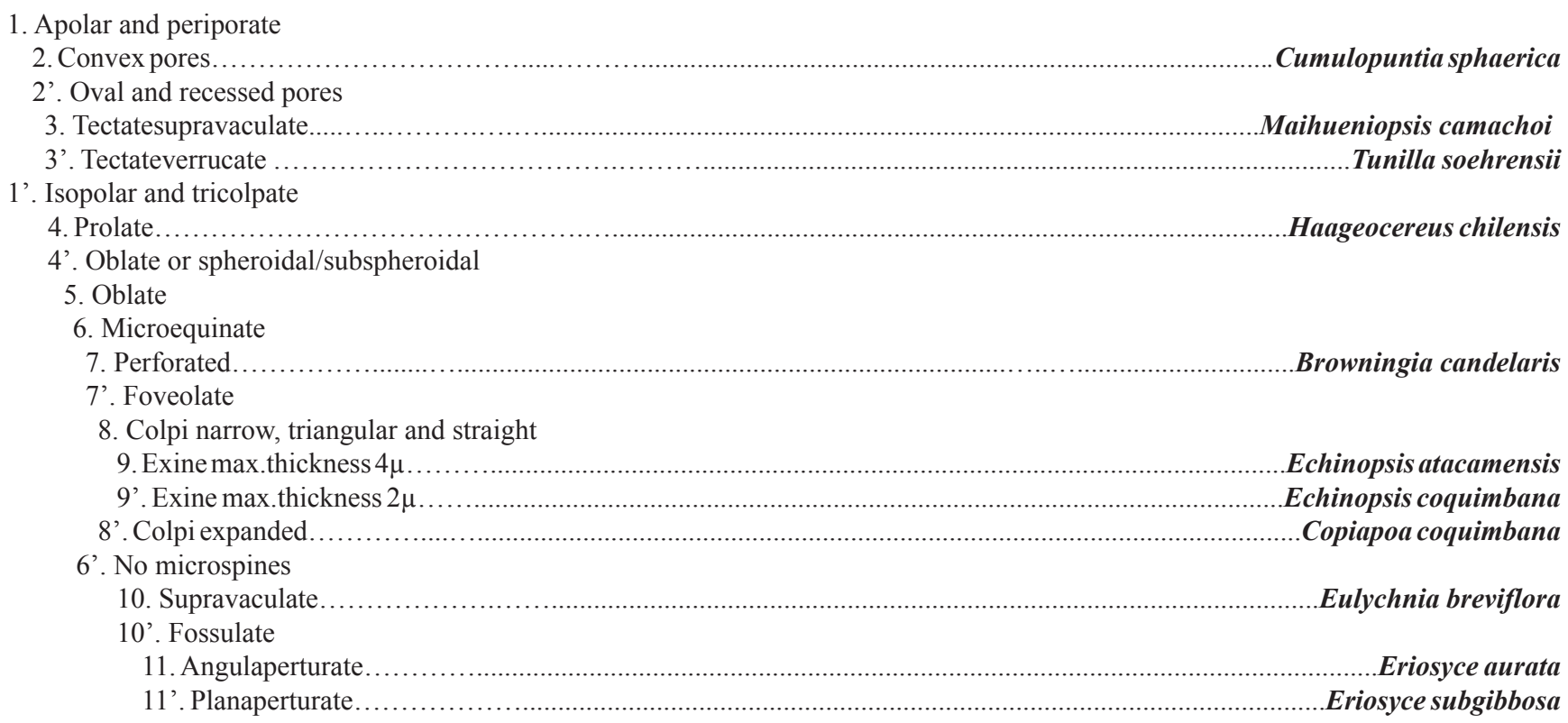


$5^{\prime}$. Spheroidal or subspheroidal

12. Colpi moderate in width $<5 \mu$ across

13. Colpi short in depth $<15 \mu$

Oreocereus hempelianus

13 '. Colpi profound in depth $>20 \mu .$.

Oreocereus leucotrichus

12 '. Colpi expanded in width $>5 \mu$ cross.

Corryocactus brevistylus

\section{ACKNOWLEDGEMENTS}

German Academic Exchange Service (DAAD), Rise Worldwide program (to FM), FONDECYT \#31305118 (to MEdP), FONDECYT \#1130279 (to AM) and CONICYT's Programa de Investigación Asociativa (PIA), Anillo Código SOC1405 (to MEdP and AM). We are grateful to Claudio Latorre for providing photographs of Maihueniopsis camachoi (Fig. 2b, c) and of Eriosyce aurata (Fig. 10b, c).

\section{REFERENCES}

Anderson, E.F. 2001. The Cactus Family. Timber Press, Portland. $776 \mathrm{pp}$.

Bennett, K.D. \& K.J. Wiluis. 2001. Pollen. In: J.P. Smol, H.J.B. Birks \& W.M.Last (eds.), Tracking Environmental Change Using Lake sediments. Terrestrial, Algal, and Siliceous Indicators, pp. 5-32. Kluwer Academic Publishers, Dordrecht, Germany.

Cuadrado, G. \& S. Garralla. 2009. Palinología de los géneros de Cactaceae, Maihuenia (Maihuenoideae) y Pereskia (Pereskioideae) de Argentina. Bomplandia 18(1): 5-12.

FAegri, K. \& L. IverSEN. 1989. Textbook of pollen analysis. 4th. Edn. John Wiley \& Sons, New York, 314 pp.

Garralla, S. \& G.A. Cuadrado. 2007. Pollen morphology of Austrocylindropuntia Backeb, Maihueniopsis Speg., Opuntia Mill. and Tephrocactus Lem. (Cactaceae, Opuntioideae) of Argentina. Review of Palaeobotany and Palynology 146: 1-17.

Garralla, S.S., G.A. Cuadrado, E.C. Lattar \& C.R. Salgado. 2013. Cactaceae. Cactoideae-Opuntioideae-Pereskioideae. En: Flora Polínica del Nordeste Argentino IV, pp. 37-46. Editorial Universitaria de la Universidad Nacional del Nordeste, Corrientes, Argentina.

Garreaud, R.D., M. Vuille \& A. Clement. 2003. The climate of the Altiplano: observed current conditions and mechanisms of past changes. Palaeogeography, Palaeoclimatology Palaeoecology 304: 1-18.

Garreaud, R.D., M. Vuille, R. Compagnucci \& J. Marengo. 2008. Present-day South America climate. Palaeogeography, Palaeoclimatology, Palaeoecology 281: 180-195.

Griffith, M.P. 2004. What Did the First Cactus Look Like? An Attempt to Reconcile the Morphological and Molecular Evidence. Taxon 53(2): 493-499.

Grosjean, M., J.F.N. Van Leeuwen, W.O. Van Der Knaap, M.A. Geyh, B. Ammann, W. Tanner, B. Messerli, L. NúÑez, B.L. Valero-Garcés \& H. Veit. 2001. A 22,000 14C year BP sediment and pollen record of climate change from Laguna Miscanti $\left(23^{\circ} \mathrm{S}\right)$, northern Chile. Global and Planetary Change 28: 35-51.

Guerrero, P.C., A.P. Durán \& H.E. Walter. 2011. Latitudinal and altitudinal patterns of the endemic cacti from the Atacama Desert to Mediterranean Chile. Journal of Arid Environments 75(11): 991-997.

Guerrero, P.C., M. Rosas, M.T.K. Arroyo \& J.J. Wiens. 2013. Evolutionary lag times and recent origin of the biota of an ancient desert (Atacama-Sechura). Proceedings of the National Academy of Sciences 110(28): 11469-11474.

Herrera, L.F. \& L.E. Urrego. 1996. Atlas de polen de plantas útiles y cultivadas de la amazonia colombiana. (Reprinted from: Estudios en la amazonia colombiana / Studies on the colombian amazonia, XI). 464 pp.

Heusser, C.J. 1971. Pollen and spores of Chile. Modern Types of Pteridophyta, Gymnospermae, and Angiospermae. The University of Arizona Press, Tucson. 167 pp.

Hoffmann, A. \& H.E. Walter. 2004. Cactáceas en la flora silvestre de Chile. Una guía para la identificación de los cactos que crecen en el país. Fundación Claudio Gay, Santiago de Chile. 307 pp.

Holmgren, M., M. Scheffer, E. Ezcurra, J.R. Gutiérrez \& G.M.J. Mohren. 2001. El Niño effects on the dynamics of terrestrial ecosystems. Trends in Ecology \& Evolution 16(2): 89-94.

Jiménez, M.A., F.M. Jaksic, J.J.Armesto, A. Gaxiola, P.L. Meserve, D.A. Kelt \& J.R. Gutiérrez. 2011. Extreme climatic events change the dynamics and invasibility of semi-arid annual plant communities. Ecology Letters 14: 1227-1235.

KuRTZ, E.B. 1948. Pollen Grain Characters of Certain Cactaceae. Bulletin of the Torrey Botanical Club 75(5): 516-522.

Kuntz, E.B. 1963. Pollen Morphology of the Cactaceae. Grana Palynologica 4(3): 367-372.

Latorre, C., J.L. Betancourt, K.A. Rylander \& J. Quade. 2002. Vegetation invasions into absolute desert: A $45000 \mathrm{yr}$ rodent midden record from the Calama-Salar de Atacama basins, northern Chile (lat $22^{\circ}-24^{\circ} \mathrm{S}$ ). Geological Society of America Bulletin 114(3): 349-366.

Latorre, C., J.L. Betancourt, K.A. Rylander, J. Quade \& O. MAtTHEI. 2003. A vegetation history from the arid prepuna of northern Chile $\left(22-23^{\circ} \mathrm{S}\right)$ over the last 13,500 years. Palaeogeography, Palaeoclimatology, Palaeoecology 194: 223-246.

Latorre, C., J.L. Betancourt, J. Quade, J.A. Rech, C. Holmgren, C. Placzek, A. Maldonado, M. Vuille \& K.A. Rylander. 2005. Late Quaternary History of the Atacama Desert. In: M. Smith \& P. Hesse (eds.), $23^{\circ}$ South: The Archaeology and Environmental History of the Southern Deserts, pp. 73-90. National Museum of Australia Press, Canberra, 
Australia.

Leuenberger, B. 1976a. Die Pollen Morphologie der Cactaceae. Dissertationes Botanicae 31: 1-321.

Leuenberger, B. 1976b. Pollen morphology of the Cactaceae. The Cactus and Succulent. Journal of Great Britain 38(4): 7994.

Maldonado, A., J.L. Betancourt, C. Latorre \& C. Villagrán. 2005. Pollen Analyses from a 50,000-Yr Rodent Midden Series in the Southern Atacama Desert (2530'S). Journal of Quaternary Science, 20(5): 493-507

Maldonado, A., C. Méndez, P. Ugalde, D. Jackson, R. Seguel \& C. LATORRE. 2010. Early Holocene climate change and the first peopling of the semiarid coast of northern Chile. Journal of Quaternary Science 25(6): 985-988.

Markgraf, V. \& H.L. D’Antoni. 1978. Pollen flora of Argentina. University of Arizona, USA. 201 pp.

Moreira-Muñoz, A. 2011. Plant Geography of Chile, Springer
Science \& Business Media, United Kingdom. 368 pp.

Mujica, M.I., C. Latorre, A. Maldonado, L. González-Silvestre, R. Pinto, R. De Pol-Holz \& C.M. Santoro. 2014. Late Quaternary climate change, relict populations and present-day refugia in the northern Atacama Desert: a case study from Quebrada La Higuera $\left(18^{\circ} \mathrm{S}\right)$. Journal of Biogeography 42 (1): 76-88. DOI:10.1111/jbi.12383.

Pinto, R. \& A. Kirberg. 2009. Cactus del Extremo Norte de Chile. AMF, Santiago de Chile. 250 pp.

Punt, W., P.P. Hoen, S. Blackmore, S. Nilsson \& A. Le Thomas. 2007. Glossary of pollen and spore terminology. Review of Palaeobotany and Palynology 143: 1-81.

Riedemann, P., G. Aldunate \& S. Teillier. 2006. Flora nativa de valor ornamental: identificación y propagación, Chile Zona Norte. Productora Gráfica Andros Limitada, Chile. 404 pp.

Señoret Espinosa, F. \& J.P. Acosta Ramos. 2013. Cactáceas endémicas de Chile. Guía de Campo. Ed. Corporación de la Madera, Concepción. 250 pp.

Recibido: 05.01.15

Aceptado: 23.09.15 